\title{
Polarized Scattering Equations for 6D Superamplitudes
}

\author{
Yvonne Geyer ${ }^{1}$ and Lionel Mason ${ }^{2}$ \\ ${ }^{1}$ Institute for Advanced Study, 1 Einstein Drive, 08540 Princeton, New Jersey, USA \\ ${ }^{2}$ Mathematical Institute, University of Oxford, Woodstock Road, Oxford OX2 6GG, United Kingdom
}

(Received 4 January 2019; published 11 March 2019)

\begin{abstract}
We introduce a spinorial version of the scattering equations, the polarized scattering equations, that incorporates spinor polarization data. They underpin new formulas for tree-level scattering amplitudes in six dimensions that directly extend to maximal supersymmetry. We find new ingredients for integrands for super Yang-Mills theory, gravity, M5 and D5 branes. We explain how the polarized scattering equations and supersymmetry representations arise from an ambitwistor string with target given by a supertwistor description of $6 \mathrm{D}$ superambitwistor space. On reduction to four dimensions, the polarized scattering equations give rise to massive analogues of the 4D refined scattering equations for amplitudes on the Coulomb branch. They give a quite distinct framework from that of Cachazo et al.; in particular, the formulas do not change character from even to odd numbers of particles.
\end{abstract}

DOI: 10.1103/PhysRevLett.122.101601

Introduction.-Six dimensions has proved to be a fertile arena both for constructing and unifying four-dimensional field theories via dimensional reduction and for studying nontrivial lower dimensional consequences of $M$ theory. In the context of scattering amplitudes, a recent exploration appeared in Refs. [1,2]. These works gave an extension of the 4D amplitude formulas arising from twistor string theories [3-7] to $6 \mathrm{D}$ for a variety of theories. Formulas for the bosonic parts of these theories had already been found in all dimensions by Cachazo, He, and Yuan (CHY) $[8,9]$, based on the scattering equations that generate $n$ points on the Riemann sphere from the null momenta of that scattered particles. These, however, lack fermion external states or supersymmetric extensions. The ambitwistor string $[10,11]$ derived these formulas from a chiral worldsheet model with target ambitwistor space, the space of complex null geodesics. This led to procedures for constructing fermionic amplitudes [12,13], but did not result in explicit formulas for $n$-point superamplitudes.

Another approach initiated in four dimensions in Ref. [14] was based on a twistor description of ambitwistor space and led to optimally compact and explicit expressions for superamplitudes. These formulas were based on the so-called refined scattering equations. These are not only refined by the number of negative helicity particles, but also incorporate the polarization data. It is this approach that we extend to six dimensions. Our formulas are motivated by a worldsheet model whose target space is a twistorial representation of $6 \mathrm{D}$

Published by the American Physical Society under the terms of the Creative Commons Attribution 4.0 International license. Further distribution of this work must maintain attribution to the author(s) and the published article's title, journal citation, and DOI. Funded by SCOAP ${ }^{3}$. superambitwistor space. Generic path integrals in this model localize on polarized scattering equations, formulated in terms of worldsheet spinors incorporating little-group indices and depending on the polarization data of the particles.

Spinors and polarization data in six dimensions. - In six dimensions, the spin group in the complex is $\operatorname{SL}(4, \mathbb{C})$ and acts on vectors as skew spinors $k^{A B}=k^{[A B]}$, where $A, B=$ $0, \ldots, 3$ are spinor indices. The metric is determined by the totally skew spinor $\varepsilon_{A B C D}=\varepsilon_{[A B C D]}$, which is also used to raise and lower skew pairs of spinor indices.

For massless particles, the little group is $\operatorname{Spin}(4)_{\mathbb{C}} \cong$ $\mathrm{SL}(2)_{+} \times \mathrm{SL}(2)_{-}$which can be seen as follows. Null momenta $k^{A B}$ with $k^{2}=k^{A B} k^{C D} \varepsilon_{A B C D}=0$ are necessarily of rank two due to the antisymmetry of the spinor indices. They can therefore be represented either by chiral or by antichiral spinors [15] as

$$
\begin{aligned}
& k^{A B}=\varepsilon^{\dot{a} \dot{b}} \kappa_{\dot{a}}^{A} \kappa_{\dot{b}}^{B}=:\left[\kappa^{A} \kappa^{B}\right], \\
& k_{A B}=\kappa_{C}^{a} \kappa_{D}^{b} \varepsilon_{a b}=:\left\langle\kappa_{A} \kappa_{B}\right\rangle .
\end{aligned}
$$

Here, $a=0,1, \dot{a}=\dot{0}, \mathrm{i}$ are the corresponding $\mathrm{SU}(2)_{ \pm}$ little group spinor indices, and we have introduced the $4 \mathrm{D}$ $\langle\cdot, \cdot\rangle$ and $[\cdot, \cdot]$ brackets for little group contractions.

A Maxwell field strength is represented by $F_{B}^{A}$, with $F_{A}^{A}=0$ because the Lie algebra of the Lorentz group is sl(4). We take momentum eigenstates with null and transverse polarization vectors and this yields

$$
F_{B}^{A}=\epsilon^{A} \epsilon_{B} .
$$

The Maxwell equations give $k_{A B} \epsilon^{A}=0=k^{A B} \epsilon_{B}$, and the polarization data is encoded in little group spinors $\epsilon_{a}$ and $\epsilon_{\dot{a}}$ with

$$
\epsilon^{A}=\epsilon_{\dot{a}} \kappa^{A \dot{a}}, \quad \epsilon_{A}=\epsilon^{a} \kappa_{A a} .
$$


The polarized scattering equations.-We take $n$ external null momenta $k_{i}, i=1, \ldots, n$ with $\sum_{i} k_{i}=0$ and associated polarization spinors $\epsilon_{i A}, \epsilon_{i}^{A}$. In the CHY formulas and the ambitwistor string, a key role is played by the meromorphic vector on the Riemann sphere with complex coordinate $\sigma \in \mathbb{C}$

$$
P(\sigma)=\sum_{i} \frac{k_{i}}{\sigma-\sigma_{i}}
$$

where $\sigma_{i}$ are $n$ marked points on $\mathbb{C P}^{1}$. Amplitudes for massless theories are given as moduli space integrals localized on the scattering equations. These are the condition that $P(\sigma)$ is a null vector for all $\sigma$ and are equivalent to $n$ equations on the $\sigma_{i}$ given by

$$
k_{i} \cdot P\left(\sigma_{i}\right)=0 .
$$

For such a null $P$, we can seek spinor-helicity factorizations $\lambda_{A}^{a}(\sigma)$ and $\lambda_{\dot{a}}^{A}(\sigma)$ of $P(\sigma)$

$$
P_{A B}=\lambda_{a A} \lambda_{B}^{a}=\frac{1}{2} \varepsilon_{A B C D} \lambda_{\dot{a}}^{C} \lambda^{D \dot{a}} .
$$

Motivated by Eq. (4), we seek $\lambda_{A a}(\sigma)$ of the form

$$
\lambda_{a A}(\sigma)=\sum_{i=1}^{n} \frac{u_{i a} \epsilon_{i A}}{\sigma-\sigma_{i}} .
$$

As a condition on the spinors, the scattering equations yield $k_{i} \cdot P=\operatorname{det}\left(\kappa_{i A}^{a}, \lambda_{A}^{b}\right)=0$. This determinant vanishes if and only if there exists nonzero $\left(u_{i}^{a}, v_{i}^{a}\right)$ so that

$$
u_{i a} \lambda_{A}^{a}\left(\sigma_{i}\right)=v_{i a} \kappa_{i A}^{a}
$$

This is scale invariant in $u$ and $v$, so we normalize with $\left\langle v_{i} \epsilon_{i}\right\rangle=1$. With this, Eq. (8) gives a deterministic system of equations in the variables $\left(\sigma_{i}, u_{i a}, v_{i a}\right)$, and provides our $6 \mathrm{D}$ polarized scattering equations

$$
\sum_{j=1}^{n} \frac{\left\langle u_{i} u_{j}\right\rangle \epsilon_{j A}}{\sigma_{i j}}-\left\langle v_{i} \kappa_{i A}\right\rangle=0 .
$$

These equations are nonsingular because $\varepsilon^{a b}$ is skew, and there exists a unique solution for each solution $\left\{\sigma_{i}\right\}$ to the unpolarized scattering equations (5). Unlike Refs. [1,2], who introduce different constructions for odd and even $n$; our polarized scattering equations do not distinguish between odd and even numbers of particles.

It is easy to see that Eq. (6) holds: there are no double poles as $u_{i}^{a} u_{i a}=0$ and

$$
\operatorname{Res}_{\sigma_{i}} \lambda(\sigma)_{A}^{a} \lambda_{B a}=\epsilon_{i[A} u_{i a} \lambda_{B]}^{a}\left(\sigma_{i}\right)=v_{i}^{b} \epsilon_{i a} \kappa_{[A}^{a} \kappa_{i B] b}=: k_{i A B}
$$

on the support of the polarized scattering equations.
The polarized scattering equations enhance the $\operatorname{SL}(2, \mathbb{C})_{\sigma}$ symmetry of the unpolarized scattering equations to a global $\mathrm{SL}(2, \mathbb{C})_{\sigma} \times \mathrm{SL}(2, \mathbb{C})_{+}$symmetry, corresponding to Möbius invariance and (chiral) little group invariance, respectively. Taking this into account, we can define a covariant measure $d \mu_{n}^{\mathrm{pol}}$ via

$$
\begin{aligned}
d \mu_{n}^{\mathrm{pol}} \equiv & \frac{\prod_{i=1}^{n} d \sigma_{i} d^{2} u_{i} d^{2} v_{i}}{\operatorname{vol~SL}(2, \mathbb{C})_{\sigma} \times \operatorname{SL}(2, \mathbb{C})_{+}} \\
& \times \prod_{i=1}^{n} \delta\left(\left\langle v_{i} \varepsilon_{i}\right\rangle-1\right) \delta^{4}\left(\left\langle u_{i} \lambda_{A}\left(\sigma_{i}\right)\right\rangle-\left\langle v_{i} \kappa_{i A}\right\rangle\right) .
\end{aligned}
$$

This measure contains $5 n$ delta functions on the $5 n-6$ variables $\left(u_{i}, v_{i}, \sigma_{i}\right)$ modulo the symmetries, leaving an overall 6D momentum-conserving delta function. The polarized scattering equations imply the scattering equations (5), and the $\left(u_{i}, v_{i}\right)$ can be explicitly integrated out to yield $d \mu_{n}^{\mathrm{pol}}=\delta^{6}\left(\sum_{i} k_{i}\right) d \mu_{n}^{\mathrm{CHY}}$, where $d \mu_{n}^{\mathrm{CHY}}$ is the standard CHY measure $\prod_{i} \delta\left[k_{i} \cdot P\left(\sigma_{i}\right)\right] d \sigma_{i} / \mathrm{SL}(2, \mathbb{C})$. For ambidextrous theories, the conjugate scattering equations $\left[\tilde{u}_{i} \lambda^{A}\left(\sigma_{i}\right)\right]-\left[\tilde{v}_{i} \kappa_{i}^{A}\right]=0$ are imposed, but not included in the measure, as in Refs. [1,2].

We will express 6D scattering amplitudes as $\int d \mu_{n}^{\mathrm{pol}} \mathcal{I}_{n}$ with, e.g.,

$$
\mathcal{I}_{n}= \begin{cases}I_{n}^{\text {spin-1 }} \mathrm{PT}(\alpha) & \text { Yang-Mills theory } \\ I_{n}^{\text {spin-1 }} \tilde{I}_{n}^{\text {spin-1 }} & \text { gravity }\end{cases}
$$

where the integrand $\mathcal{I}_{n}$ is a meromorphic function of the scattering data and the variables $\left(\sigma_{i}, u_{i a}, v_{i a}\right)$ carrying $\operatorname{SL}(2, \mathbb{C})_{\sigma}$ weight 2 . Here $\operatorname{PT}(\alpha)=\prod\left(\sigma_{\alpha(i)}-\sigma_{\alpha(i+1)}\right)^{-1}$ is the Parke-Taylor factor associated to the permutation $\alpha$ and $I_{n}^{\text {spin-1 }}$ is a function of Maxwell polarization data that is gauge and permutation invariant on each solution to the polarized scattering equations. The CHY Pfaffians are possible choices for $I_{n}^{\text {spin-1 }}$, but we will have more appropriate alternatives.

Supersymmetry.-In six dimensions, $(N, \tilde{N})$ supersymmetry possesses an $\operatorname{Sp}(N) \times \operatorname{Sp}(\tilde{N}) R$-symmetry group for which we introduce indices $I=1, \ldots, 2 N$, and $\dot{I}=$ $\dot{1}, \ldots, \dot{2} \tilde{N}$. On momentum eigenstates with momentum $k_{A B}$, the supersymmetry generators $Q_{A I}$ and $Q_{\dot{I}}^{A}$ satisfy [16]

$$
\left\{Q_{A I}, Q_{B J}\right\}=k_{A B} \Omega_{I J}, \quad\left\{Q_{\dot{I}}^{A}, Q_{j}^{B}\right\}=k^{A B} \Omega_{\dot{I} J},
$$

where $\Omega_{I J}$ and $\Omega_{\dot{I} J}$ are the $R$-symmetry symplectic metrics. The supersymmetry generators thus reduce to the little group as

$$
Q_{A I}=\kappa_{A}^{a} Q_{a I}, \quad Q_{\dot{I}}^{A}=\kappa_{\dot{a}}^{A} Q_{\dot{I}}^{\dot{a}} .
$$

A key example is $(1,1)$ super Yang-Mills theory, with the supermultiplet 


$$
\mathcal{F}:=\left(F_{A}^{B}, \psi_{I}^{A}, \tilde{\psi}_{A \dot{I}}, \phi_{I \dot{I}}\right) .
$$

On momentum eigenstates with null momentum $k_{A B}, Q_{C J}$ acts on this multiplet by

$$
Q_{C J} \mathcal{F}=\left(k_{A C} \psi_{J}^{B}, \Omega_{J I} F_{C}^{A}, k_{A C} \phi_{J I}, \Omega_{J I} \tilde{\psi}_{I C}\right) .
$$

To construct an on-shell superspace, half of the generators need to be selected as supermomenta. Two mechanisms have been discussed in the literature [17], manifesting either little-group or $R$ symmetry. While the former has been employed successfully in recent work on $6 \mathrm{D}$ scattering amplitudes for a variety of theories [1,2], the latter is more natural from the perspective of the ambitwistor string [18], and will be the formulation we work with here. The two approaches are of course related by appropriate Grassmann Fourier transforms.

In the context of the polarized scattering equations, $\epsilon_{a}, \epsilon_{\dot{a}}$, and $v_{a}, v_{a}$ give a natural choice for breaking the little-group symmetry. Using this, the full supermultiplet can be parametrized by supermomenta $\left(q_{I}, \tilde{q}_{\dot{I}}\right)$ by imposing the relations

$$
Q_{a I} \mathcal{F}(q, \tilde{q})=\left(v_{a} q_{I}+\epsilon_{a} \Omega_{I J} \frac{\partial}{\partial q_{J}}\right) \mathcal{F}(q, \tilde{q}) .
$$

The full super Yang-Mills multiplet can be obtained from the pure gluon state $\mathcal{F}(0,0)=\left(\epsilon_{A} \epsilon^{B}, 0,0,0\right)$ in this representation as

$$
\begin{aligned}
\mathcal{F}\left(q_{I}, \tilde{q}_{\dot{I}}\right)= & \left(\left(\epsilon_{A}+q^{2}\left\langle v \kappa_{A}\right\rangle\right)\left(\epsilon^{B}+\tilde{q}^{2}\left\langle v \kappa^{B}\right\rangle\right),\right. \\
& \left.q_{I}\left(\epsilon^{A}+\tilde{q}^{2}\left\langle v \kappa^{A}\right\rangle\right), \tilde{q}_{\dot{I}}\left(\epsilon_{A}+q^{2}\left\langle v \kappa_{A}\right\rangle\right), q_{I} \tilde{q}_{\dot{I}}\right) .
\end{aligned}
$$

While this supersymmetry representation is dynamic and particle specific, the full supersymmetry generator for $n$ particles is still defined by the sum $Q_{A I}=\sum_{i=1}^{n} Q_{i A I}$. Superamplitudes are supersymetrically invariant and so are annihilated by these $Q_{A I}$. We will see below that this implies that the total dependence on the supermomenta is encoded in a simple exponential factor $e^{F}$, with $F=F_{N}+\tilde{F}_{\tilde{N}}$, where

$F_{N}=\sum_{i<j} \frac{\left\langle u_{i} u_{j}\right\rangle q_{i I} q_{j}^{I}}{\sigma_{i j}}, \quad \tilde{F}_{\tilde{N}}=\sum_{i<j} \frac{\left[\tilde{u}_{i} \tilde{u}_{j}\right] \tilde{q}_{i I} \tilde{q}_{j}^{I}}{\sigma_{i j}}$.

For $\mathcal{N}=(1,1)$ super Yang-Mills theory, for example, this exponential factor becomes $\exp F^{\mathrm{YM}}=\exp \left(F_{1}+\tilde{F}_{1}\right)$. Below, we will also provide a geometric derivation of the factor $e^{F_{N}}$ from a worldsheet model.

In general, given a scattering amplitude of the form (11) for the top states of the multiplet of an $\mathcal{N}=(N, \tilde{N})$ theory, the fully supersymmetric amplitude is given by

$$
\mathcal{A}_{n}=\int d \mu_{n}^{\mathrm{pol}} \mathcal{I}_{n} e^{F}
$$

This is invariant under supersymetry because

$Q_{A I} e^{F}=\left(\sum_{i}\left\langle v_{i} \kappa_{i A}\right\rangle q_{i I}-\sum_{i, j} \frac{\left\langle u_{i} u_{j}\right\rangle \epsilon_{i A}}{\sigma_{i j}} q_{j I}\right) e^{F}=0$,

on the support of the polarized scattering equations. Similarly $Q_{\dot{I}}^{A} e^{F}=0$. Conversely, given an integrand $\mathcal{I}_{n}$ for the top states of a multiplet, (19) is the unique supersymmetric completion using the supersymmetry representation (16), as can be verified using supersymmetric Ward identities.

Integrands. -We now construct the integrands $\mathcal{I}_{n}$ for SYM theory, supergravity, D5 theory, and $M 5$ theory. For the ambidextrous spin one contribution, define an $n \times n$ matrix $H$ by

$$
H_{i j}= \begin{cases}\frac{\epsilon_{i A} \epsilon_{j}^{A}}{\sigma_{i j}} & i \neq j, \\ H_{i i} & \end{cases}
$$

where $H_{i i}$ is defined equivalently by

$$
\lambda_{a A}\left(\sigma_{i}\right) \epsilon_{i}^{A}=-u_{i a} H_{i i}, \quad \lambda^{\dot{a} A}\left(\sigma_{i}\right) \epsilon_{i A}=-u_{i}^{\dot{a}} H_{i i} .
$$

The left-hand side is a multiple of $u_{i a}$ (or $u_{i}^{\dot{a}}$ ) due to the scattering equation and the identity $k^{A B} \kappa_{A}^{a}=0$.

On the polarized scattering equations, the determinant det $H$ vanishes because $H$ has co-rank 2 due to

$$
\sum_{i} u_{i a} H_{i j}=\lambda_{a A}\left(\sigma_{j}\right) \epsilon_{j}^{A}+u_{j a} H_{j j}=0 .
$$

The first term follows from the definition (7) of $\lambda_{a A}$ and the second equality from Eq. (22). Similarly, $\sum_{j} H_{i j} u_{j \dot{a}}=0$. These identities nevertheless imply that $H$ has a welldefined reduced determinant

$$
\operatorname{det}^{\prime} H:=\frac{\operatorname{det}\left(H_{\left[j_{1} j_{2}\right]}^{\left[i_{1} i_{2}\right]}\right)}{\left\langle u_{i_{1}} u_{i_{2}}\right\rangle\left[u_{j_{1}} u_{j_{2}}\right]} .
$$

Here, $H_{\left[i_{2} j_{3}\right]}^{\left[i_{1} j_{1}\right]}$ denotes the matrix $H$ with the rows $i_{1}, i_{2}$ and columns $j_{1}, j_{2}$ deleted, and $\operatorname{det}^{\prime} H$ is well defined in the sense that the Eq. (24) is invariant under permutations of particle labels, and thus independent of the choice of $i_{1,2}, j_{1,2}$.

To see this, we extend the argument of Ref. [19] to reduced determinants of general matrices. Since the $n \times n$ matrix $H_{i}^{j}$ satisfies $\sum_{i} u_{a}^{i} H_{i}^{j}=0$ and $\sum_{j} H_{i}^{j} \tilde{u}_{j}^{\dot{b}}=0$, we have

$$
\varepsilon^{i_{1} \ldots i_{n}} \varepsilon_{j_{1} \ldots j_{n}} H_{i_{3}}^{j_{3}} \ldots H_{i_{n}}^{j_{n}}=\operatorname{det}^{\prime}(H)\left\langle u^{\left[i_{1}\right.} u^{\left.i_{2}\right]}\right\rangle\left[\tilde{u}_{\left[j_{1}\right.} \tilde{u}_{j_{2}}\right]
$$

for some $\operatorname{det}^{\prime} H$ because the $u$ 's and $\tilde{u}$ 's span the left and right kernel of $H$. The definition (24) then follows. 
The reduced determinant $\operatorname{det}^{\prime} H$ is manifestly gauge invariant in all particles, carries $\operatorname{SL}(2, \mathbb{C})_{\sigma}$ weight -2 , as expected for a half-integrand $I^{\text {spin-1 }}$ and is equally valid for even and odd numbers of external particles. On the support of the polarized scattering equations, it can be verified using factorization that $\operatorname{det}^{\prime} H$ is equal to the CHY halfintegrand $\mathrm{Pf}^{\prime} M$.

Another important building block, relevant for the D5 and $M 5$ theory, is the skew matrix $A$, familiar from the CHY formulas [8,9], with

$$
A_{i j}=\frac{k_{i} \cdot k_{j}}{\sigma_{i j}} .
$$

Again, the Pfaffian $\operatorname{Pf} A$ vanishes on the scattering equations (5), but the reduced Pfaffian $\operatorname{Pf}^{\prime} A=$ $\left[(-1)^{i+j} / \sigma_{i j}\right] \mathrm{Pf} A_{i j}^{i j}$ is well defined and nonzero for even numbers of particles $[8,9]$.

The final two ingredients are constructed exclusively using the variables $\left(\sigma_{i}, u_{i a}, \tilde{u}_{i \dot{a}}\right)$, and are only needed for M5 branes. These only lead to amplitudes with even numbers of particles. Consider any partition of the particle labels into two sets [20] $Y$ and $\bar{Y}$, with $|Y|=|\bar{Y}|=n / 2$, and define $n / 2 \times n / 2$ matrices $U, \tilde{U}$ and $X$ by

$$
U_{i r}=\frac{\left\langle u_{i} u_{r}\right\rangle}{\sigma_{i r}}, \quad \tilde{U}_{i r}=\frac{\left[\tilde{u}_{i} \tilde{u}_{r}\right]}{\sigma_{i r}}, \quad X_{i r}=\frac{1}{\sigma_{i r}},
$$

for $i \in Y$ and $r \in \bar{Y}$. Schouten identities guarantee that the ratios

$$
\frac{\operatorname{det} \tilde{U}}{\operatorname{det} U}, \quad \frac{\operatorname{det} X}{\operatorname{det}^{2} \mathrm{U}}, \quad \frac{\operatorname{det} X}{\operatorname{det} U \operatorname{det} \tilde{U}},
$$

are independent of the choice of partition into $Y$ and $\bar{Y}$. Then the combinations

$$
\frac{\operatorname{det} X}{\operatorname{det}^{2} U} \operatorname{Pf}^{\prime} A, \quad \frac{\operatorname{det} X}{\operatorname{det} U \operatorname{det} \tilde{U}} \operatorname{Pf}^{\prime} A=\operatorname{det}^{\prime} H,
$$

form chiral half-integrands, independent of the partition $Y, \bar{Y}$. The latter equality follows from comparison to the $\mathrm{CHY}$ integrands when restricted to a scalar subsector.

At this point, we have all the ingredients to present the integrands of $\mathcal{N}=(1,1)$ super Yang-Mills theory, $\mathcal{N}=$ $(2,2)$ supergravity, $\mathcal{N}=(1,1) D 5$ theory, and $\mathcal{N}=(2,0)$ M5 theory;

Super Yang-Mills theory: $\quad \operatorname{PT}(\alpha) \operatorname{det}^{\prime} H e^{F_{1}+\tilde{F}_{1}}$.

$$
\begin{array}{ll}
\text { Supergravity: } & \operatorname{det}^{\prime} H \operatorname{det}^{\prime} \tilde{H} e^{F_{2}+\tilde{F}_{2}} . \\
\text { D5 - branes: } & \operatorname{det}^{\prime} A \operatorname{det}^{\prime} H e^{F_{1}+\tilde{F}_{1}} . \\
\text { M5 - branes: } & \frac{\operatorname{det} X}{\operatorname{det}^{2} \mathrm{U}}\left(\operatorname{Pf}^{\prime} A\right)^{3} e^{F_{2}} .
\end{array}
$$

It is easily seen that the resulting superamplitudes are $\operatorname{SL}(2, \mathbb{C})_{\sigma} \times \operatorname{SL}(2, \mathbb{C})_{ \pm}$invariant. As discussed above, the SYM and supergravity amplitudes are gauge invariant, and the supergravity amplitudes are permutation invariant. Moreover, the super Yang-Mills and supergravity amplitudes satisfy color-kinematics duality, as evident from the form of their amplitudes, and the M5 amplitudes are manifestly chiral. We have checked that all amplitudes reproduce the correct three-point amplitudes, factorize correctly, and that the supergravity and super Yang-Mills amplitudes reduce to the well-known expressions $[3,5,6]$ in four dimensions (which, in particular, gives a direct check on four- and five-point amplitudes).

Ambitwistor strings.-In the following, we develop a twistorial 6d ambitwistor string-a chiral 2D CFT whose target space is ambitwistor space $\mathbb{A}$, the space of complex light rays in complexified $6 \mathrm{D}$ space-time. This provides a geometric interpretation of the polarized scattering equations and the exponential supersymmetry factors.

We focus here on the model using a chiral representation of super ambitwistor space. However, amplitudes in ambidextrous theories require both the chiral and antichiral supertwistors. Since these are alternative coordinates on the same space, they are related by nontrivial constraints. Implementing these constraints at the level of the worldsheet model, as well as adding "worldsheet matter" systems giving rise to the integrands $\mathcal{I}_{n}$, is beyond the scope of this Letter.

Ambitwistor space.-In six dimensions $\mathbb{A}$ has a canonical twistor representation as follows. Extend space-time to superspace to include fermionic coordinates $\theta^{A I}$ with $I=$ $1, \ldots, 2 N$. Projective supertwistor space is then given by the quadric in $\mathbb{C P}^{7 \mid 2 N}$ with homogeneous coordinates [21-24]

$$
\mathcal{Z}=\left(\lambda_{A}, \mu^{A}, \eta^{I}\right)
$$

satisfying

$$
\mathcal{Z} \cdot \mathcal{Z}:=\mu^{A} \lambda_{A}+\mu^{A} \lambda_{A}+\Omega_{I J} \eta^{I} \eta^{J}=0 .
$$

A twistor $\mathcal{Z}$ determines a totally null self-dual $3 \mid 6 N$-plane in space-time via the incidence relations

$$
\mu^{A}=x^{A B} \lambda_{B}+\theta^{I A} \theta_{I}^{B} \lambda_{B}, \quad \eta^{I}=\theta^{I A} \lambda_{A} .
$$

Generically such three-planes are disjoint, but intersect along a null geodesic when the corresponding twistors satisfy $\mathcal{Z}^{0} \cdot \mathcal{Z}^{1}=0$. Superambitwistor space $\mathbb{A}$ can thus be represented by a pair of twistors, i.e., $\mathcal{Z}^{a}=\left(\lambda_{A}^{a}, \mu^{A a}\right)$ subject to $\mathcal{Z}^{a} \cdot \mathcal{Z}^{b}=0$ modulo the symmetry group $\mathrm{SL}(2, \mathbb{C})_{+}$acting on the $a$ index.

Supersymmetric ambitwistor strings.-In the superambitwistor string, the supertwistors are taken to be worldsheet spinors, with the chiral action [25]

$$
S=\int_{\Sigma} \epsilon_{a b} \mathcal{Z}^{a} \bar{\partial} \mathcal{Z}^{b}+A_{a b} \mathcal{Z}^{a} \cdot \mathcal{Z}^{b}
$$


In this action, we have already gauge-fixed worldsheet gravity, leading to the usual $\operatorname{SL}(2, \mathbb{C})_{\sigma}$ quotient in the measure. The field $A_{a b}=A_{(a b)} \in \Omega^{(0,1)}$ gauges the little group $\operatorname{SL}(2, \mathbb{C})_{+}$symmetry, reducing the target space from two copies of twistor space to ambitwistor space. The ambitwistor string is a chiral CFT that becomes free and linear after BRST gauge-fixing $A_{a b}=0$.

Vertex operators in the ambitwistor string are built from ambitwistor representatives for space-time fields, which are Dolbeault cohomology classes in $H^{1}(P \mathbb{A})$. In the bosonic case, they have to reduce to $\int_{\Sigma} w \delta\left(k_{i} \cdot P\right) e^{i k_{i} \cdot x}$, where $w$ is some worldsheet matter that depends on the polarization data. This is achieved by

$$
\int d^{2} u d^{2} v \bar{\delta}(\langle v \varepsilon\rangle-1) \delta^{4}\left(\left\langle u \lambda_{A}\right\rangle-\left\langle v \kappa_{A}\right\rangle\right) w \mathrm{e}^{i u^{a}\left(\mu_{a}^{A} \epsilon_{A}+\eta_{a}^{I} q_{I}\right)} .
$$

This vertex operator has cohomology degree one since one delta function remains after integrating out the $\left(u_{i}, v_{i}\right)$. Moreover, by the discussion after Eq. (9), this remaining delta function is a multiple of $\delta\left(k_{i} \cdot P\right)$. To see that the exponent gives the correct supersymmetric completion, first observe that the defining relations (16) for supermomenta $\left(q_{I}, \tilde{q}_{\dot{I}}\right)$ are solved by $\exp \left(i k \cdot x+q_{I} \theta^{I A} \kappa_{A}^{a} v_{a}+\Omega_{I J} \theta^{I A} \theta^{J B} k_{A B}\right)$. Then the incidence relations and polarized scattering equations identify the exponent with that in Eq. (34).

Scattering equations and supersymmetry.-This general form for the vertex operator is sufficient to derive the polarized scattering equations and the supersymmetry factor $e^{F_{N}}$ from the ambitwistor string path integral. Consider the correlation function of $n$ vertex operators, indexed by $i=1, \ldots, n$. The $\mathcal{Z}$ path integration in this correlator can be performed by taking the exponentials into the action to yield sources for $\lambda_{a A}$ and $\eta^{I a}$;

$$
\bar{\partial}\left(\lambda_{a A}, 2 \eta^{I a}\right)=\sum_{i}\left(\epsilon_{i A} u_{i a}, u_{i}^{a} q_{i}^{I}\right) \bar{\delta}\left(\sigma-\sigma_{i}\right) .
$$

Since $\lambda$ and $\eta$ are worldsheet spinors, this has the unique solution

$$
\left(\lambda_{A}^{a}(\sigma), \eta^{I a}\right)=\sum_{i=1}^{n}\left(\frac{u_{i}^{a} \epsilon_{i A}}{\sigma-\sigma_{i}}, \frac{u_{i}^{a} q_{i}^{I}}{2\left(\sigma-\sigma_{i}\right)}\right),
$$

justifying our ansatz in Eq. (7). The delta functions then impose the polarized scattering equations (9) irrespective of the worldsheet matter, whose correlators give rise to the integrands $\mathcal{I}_{n}$. The remaining contribution to the amplitude is via the exponential factor in Eq. (34) which reduces, after substituting the solution (36), to give

$$
\exp \left(F_{N}\right)=\exp \left(\sum_{i<j} \frac{\left\langle u_{i} u_{j}\right\rangle q_{i I} q_{j}^{I}}{\sigma_{i j}}\right) .
$$

Coulomb branch of $\mathcal{N}=4$ SYM theory.-The 6D $(1,1)$ SYM amplitudes (30a) can be used to obtain amplitudes for $4 \mathrm{D} \mathcal{N}=4$ SYM theory on the Coulomb branch $[1,26]$. On reduction to four dimensions, the spinor index can be broken up into two-component 4D spinor indices as $A=$ $(\alpha, \dot{\alpha})$ and the two $\mathrm{SU}(2)$ factors of the 6D little group can both be identified with the 4D massive one, so $a=\dot{a}$. The 4D massive kinematic data are $\left(\lambda_{\alpha a}, \tilde{\lambda}_{\dot{\alpha} a}\right)$, where $a=0,1$ is the 4D massive little group index [27], and

$$
\begin{aligned}
& \lambda_{\alpha a} \tilde{\lambda}_{\dot{\alpha} b} \epsilon^{a b}=k_{\alpha \dot{\alpha}}, \\
& \lambda_{\alpha a} \lambda_{\beta b} \epsilon^{a b}=M \epsilon_{\alpha \beta}, \quad \tilde{\lambda}_{\dot{\alpha} a} \tilde{\lambda}_{\dot{\beta} b} \epsilon^{a b}=\tilde{M} \epsilon_{\dot{\alpha} \dot{\beta}} .
\end{aligned}
$$

This embeds into the 6D spinor-helicity formalism, reinterpreting some of the spinor components of the momenta as masses, as

$$
\kappa_{a A}=\left(\begin{array}{c}
\lambda_{\alpha a} \\
\tilde{\lambda}_{a}^{\dot{\alpha}}
\end{array}\right), \quad \kappa_{\dot{a}}^{A}=\kappa_{a}^{A}=\left(\begin{array}{c}
\lambda_{a}^{\alpha} \\
\tilde{\lambda}_{\dot{\alpha} a}
\end{array}\right) .
$$

with $M \tilde{M}=m^{2}$. The reduction to massless kinematics follows by setting $\lambda_{\alpha, 0}=\tilde{\lambda}_{\dot{\alpha}, 1}=0$.

For $4 \mathrm{D} \mathcal{N}=4-\mathrm{SYM}$ theory, masses are introduced by giving some of the scalars a nonzero vev $\phi_{0}$ that spontaneously breaks the gauge group from $\mathrm{U}(N+M)$ to $\mathrm{U}(N) \times \mathrm{U}(M)$. This gives a mass $m=g_{\mathrm{YM}} \phi_{0}$ to the offdiagonal gauge bosons, now bifundamentals under both gauge groups.

Denote 4D little group contractions by round brackets, e.g., $\left(u_{i} u_{j}\right):=u_{i a} u_{j}^{a}$ and reserve now the angle and square bracket for the usual 4D spinor-helicity contractions. In this notation, the polarized massive $4 \mathrm{D}$ scattering equations are given by

$$
\sum_{j} \frac{\left(u_{i} u_{j}\right)}{\sigma_{i j}} \epsilon_{j \alpha}=v_{i a} \lambda_{i \alpha}^{a}, \quad \sum_{j} \frac{\left(u_{i} u_{j}\right)}{\sigma_{i j}} \tilde{\epsilon}_{j}^{\dot{\alpha}}=v_{i a} \tilde{\lambda}_{i}^{\dot{\alpha} a} .
$$

Both the chiral and the antichiral $6 \mathrm{D}$ polarized scattering equations descend to the same massive $4 \mathrm{D}$ scattering equations.

The matrix $H$ used to define the SYM integrand also reduces straightforwardly, with entries

$$
H_{i j}^{\mathrm{CB}}=\frac{\left\langle\epsilon_{i} \epsilon_{j}\right\rangle+\left[\tilde{\epsilon}_{i} \tilde{\epsilon}_{j}\right]}{\sigma_{i j}} .
$$

Its reduced determinant is defined as before as $\operatorname{det}^{\prime} H^{\mathrm{CB}}=\left(u_{i_{1}} u_{i_{2}}\right)^{-1}\left(u_{j_{1}} u_{j_{2}}\right)^{-1} \operatorname{det} H_{\left[j_{1} j_{2}\right]}^{\mathrm{CB}}\left[i_{1} i_{2}\right]$. The full scattering amplitude of $\mathcal{N}=4 \mathrm{SYM}$ theory on the Coulomb branch is then given by

$$
\mathcal{A}_{n}^{\mathrm{CB}}=\int d \mu_{n}^{\mathrm{CB}} \mathrm{PT}(\alpha) \operatorname{det}^{\prime} H^{\mathrm{CB}} e^{F_{1}+\tilde{F}_{1}},
$$


TABLE I. Combinations of half-integrands.

\begin{tabular}{lcccc}
\hline \hline & PT & $\operatorname{det}^{\prime} A$ & $\operatorname{det}^{\prime} H e^{F_{1}+\tilde{F}_{1}}$ & {$\left[\left(\operatorname{Pf}^{\prime} A \operatorname{det} X e^{F_{2}}\right) /\left(\operatorname{det}^{2} U\right)\right]$} \\
\hline PT & Bi-adj & NLSM & $\mathcal{N}=(1,1) \operatorname{SYM}$ & $\mathcal{N}=(2,0)$ \\
$\operatorname{det}^{\prime} A$ & & Galileon & $\mathcal{N}=(1,1) D 5$ & $\mathcal{N}=(2,0) \mathrm{M} 5$ \\
$\operatorname{det}^{\prime} H e^{F_{1}+\tilde{F}_{1}}$ & & $\mathcal{N}=(2,2)$ sugra & $\mathcal{N}=(3,1)$ \\
{$\left[\left(\operatorname{Pf}^{\prime} A \operatorname{det} X e^{F_{2}}\right) /\left(\operatorname{det}^{2} U\right)\right]$} & & & & $\mathcal{N}=(4,0)$ \\
\hline \hline
\end{tabular}

where the measure $d \mu_{n}^{\mathrm{CB}}$ on the Coulomb branch is given by Eq. (10) but with the delta functions now imposing the massive 4D polarized scattering equations (40). The further reduction to massless amplitudes immediately gives the gauge and gravity formulas of Ref. [14] based on the refined scattering equations.

Discussion.-Our new formulae are represented in double-copy form in Table 1. Major distinctions between our work and that of Ref. [1] are that there is now no artificial distinction between even and odd particles in the polarized scattering equations, and there are tentative worldsheet models whose path integrals yield much of our framework. The distinction between even and odd particle numbers in our work is physical in the integrands for $M 5$ and $D 5$ branes where the Pfaffians $\operatorname{Pf}^{\prime} A$ of skew $n \times n$ matrices naturally vanish when $n$ is odd.

There are many directions for future investigation. A natural task is to find suitable worldsheet matter systems to provide integrands for the bare ambitwistor-string models described here. Provided that the constraints relating chiral and antichiral supertwistors have been implemented in the worldsheet model, there is a natural candidate for the matter system giving rise to the $\operatorname{det}^{\prime} H$ factors, constructed from fermionic fields $\left(\rho_{A}, \rho^{A}\right) \in K^{1 / 2} \otimes\left(\mathbb{S}_{A}, \mathbb{S}^{A}\right)$ and $\left(B_{a}, B^{\dot{a}}\right) \in \Omega^{0,1} \otimes\left(\mathbb{S}_{a}, \mathbb{S}^{\dot{a}}\right) ;$

$$
S_{\rho}=\int_{\Sigma} \rho_{A} \bar{\partial} \rho^{A}+\tilde{B}^{\dot{a}} \lambda_{\dot{a}}^{A} \rho_{A}+B_{a} \lambda_{A}^{a} \rho^{A} .
$$

A different question concerns what other theories might be constructed using the polarized scattering equations. One immediate observation is that new formulas can be constructed by combining the hal-integrands introduced in this Letter given in the last column of Table 1. While the interpretation of these new formulas remains unclear, they are well defined and manifestly supersymmetric, and thus worth studying in their own right.

We would like to thank the authors of Ref. [1] for freely discussing their work when it was in its early stages and sharing drafts. Y. G. gratefully acknowledges support from the National Science Foundation Grant No. PHY-1606531 and the Association of Members of the Institute for Advanced Study (AMIAS). L. M. is grateful to the EPSRC for support under Grant No. EP/M018911/1.
[1] F. Cachazo, A. Guevara, M. Heydeman, S. Mizera, J. H. Schwarz, and C. Wen, J. High Energy Phys. 09 (2018) 125.

[2] M. Heydeman, J. H. Schwarz, and C. Wen, J. High Energy Phys. 12 (2017) 003.

[3] R. Roiban, M. Spradlin, and A. Volovich, Phys. Rev. D 70, 026009 (2004).

[4] F. Cachazo and D. Skinner, Phys. Rev. Lett. 110, 161301 (2013).

[5] E. Witten, Commun. Math. Phys. 252, 189 (2004).

[6] N. Berkovits, Phys. Rev. Lett. 93, 011601 (2004).

[7] D. Skinner, arXiv:1301.0868.

[8] F. Cachazo, S. He, and E. Y. Yuan, Phys. Rev. Lett. 113, 171601 (2014).

[9] F. Cachazo, S. He, and E. Y. Yuan, J. High Energy Phys. 07 (2015) 149.

[10] L. Mason and D. Skinner, J. High Energy Phys. 07 (2014) 048.

[11] E. Casali, Y. Geyer, L. Mason, R. Monteiro, and K. A. Roehrig, J. High Energy Phys. 11 (2015) 038.

[12] T. Adamo, E. Casali, and D. Skinner, J. High Energy Phys. 04 (2014) 104.

[13] N. Berkovits, J. High Energy Phys. 03 (2014) 017.

[14] Y. Geyer, A. E. Lipstein, and L. J. Mason, Phys. Rev. Lett. 113, 081602 (2014).

[15] C. Cheung and D. O'Connell, J. High Energy Phys. 07 (2009) 075.

[16] In the following, we suppress the particle index $i$ for readability.

[17] Y.-t. Huang and A. E. Lipstein, J. High Energy Phys. 10 (2010) 007.

[18] I. Bandos, J. High Energy Phys. 09 (2014) 086.

[19] F. Cachazo, L. Mason, and D. Skinner, SIGMA 10, 051 (2014).

[20] For scalar amplitudes, this corresponds to a partition into two non-self-interacting scalar sectors.

[21] R. Penrose and W. Rindler, Spinors and Space-Time: Spinor and Twistor Methods in Space-Time Geometry (Cambridge University Press, Cambridge, England, 1986), Vol. 2, p. 501.

[22] L. J. Mason, R. A. Reid-Edwards, and A. Taghavi-Chabert, J. Geom. Phys. 62, 2353 (2012).

[23] T. Chern, arXiv:0906.0657.

[24] L. J. Mason and R. A. Reid-Edwards, arXiv:1212.6173.

[25] This is equivalent to the action in a vector representation [10] as can be seen using $P_{A B}=\lambda_{A a} \lambda_{B}^{a}$ and the incidence relations.

[26] Z. Bern, J. J. Carrasco, T. Dennen, Y.-t. Huang, and H. Ita, Phys. Rev. D 83, 085022 (2011).

[27] N. Arkani-Hamed, T.-C. Huang, and Y.-t. Huang, arXiv: 1709.04891. 